\title{
Design, Analysis, and Optimization of LCD Backlight Unit using Ray Tracing Simulation
}

\author{
Joonsoo Choi ${ }^{1}$, Kwang-Soo Hahn ${ }^{1}$, Heekyung Seo ${ }^{1}$, Seong-Cheol Kim ${ }^{2}$ \\ ${ }^{1}$ School of Computer Science, Kookmin University, Republic of Korea \\ \{jschoi, kshahn\}@kookmin.ac.kr \\ \{hkseo\}@cs-mail.kookmin.ac.kr \\ ${ }^{2}$ School of Electrical Engineering and Computer Science, \\ Seoul National University, Republic of Korea \\ sckim@maxwell.snu.ac.kr
}

\begin{abstract}
The design of BLU for LCD devices, whose goal is to achieve uniform illumination and high luminance across the LCD surface, requries an assistance of illumination design programs. The goal of this paper is to develop a design and analysis tool to model an efficient BLU. The rendering techniques traditionally used in the field of computer graphics are the usual tools of choice to analyze BLU. An analysis method based on Monte Carlo photon tracing to evaluate the optical performance of BLU is presented. An optimization technique based on direct search method, a simplex method by Nelder and Mead, to achieve an optimal uniform illumination is also discussed.
\end{abstract}

\section{Introduction}

A liquid crystal display (LCD) is a standard display device for hand-held systems such as notebook computer, PDA, cellular phone, etc. Since liquid crystals are not lightemitting materials, backlight unit (BLU) which is usually placed behind the LCD panel is used for an LCD system as a light source. A typical BLU consists of a lightguiding plate (LGP) and a light source which is located at edges of the LGP to minimize the thickness of the unit. LGP is an optically transparent plate which is rectangular or wedge in shape. Radiated light from the source is conducted into the LGP and is guided inside the LGP based on the principle of total internal reflection. The light is reflected by an array of diffusive ink dots and emitted out the front face of the LGP. The emanated light is dispersed by the diffusing sheet and collimated by prism sheets before it eventually reaches the viewer's eye.

The design of BLU, whose goal is to maximize the light intensity and control the light distribution on the front face of LGP, requires the assistance of illumination design programs. The rendering techniques traditionally used in the field of computer graphics are the usual tools of choice to analyze BLU $[1,2]$. We describe an analysis method based on Monte Carlo photon tracing [3, 4] to evaluate the optical performance of BLU. 
One of the design challenges of BLU is to achieve a proper uniformity of the emanated light on the surface of LGP. To achieve the uniformity, the arrangement and a density or fill factor gradation of the diffusing ink dots are controlled. Usually the diameters of ink dots increase along the propagation direction of light in the LGP. It is difficult to control the density of ink dots by manual operation so that it contributes to the generation of illumination uniformity on the front face of BLU. Therefore optimization technique to automatically compute the best values for the variable parameters is needed to achieve an optimal uniform illumination. The optimization in the field of designing optical illumination devices is an immature field and it is hardly to find a design tool that implements a general optimization algorithm [5].

The objective function in the optimization problem to achieve uniform illumination has some characteristics that are unattractive to apply standard powerful optimization techniques like Newton-based and quasi-Newton methods [6]. Furthermore computation of the objective function is very expensive and time-consuming when the number of photons generated to simulate the performance of BLU is very large. Therefore it is desirable to find an optimization method locating a minimizer in as few function evaluations as possible. Direct search method is a potential candidate for the optimization since it uses only function values and does not require a gradient. The simplex method devised by Nelder and Mead [7] which is widely used method in the class of direct search method is selected to implement the optimization. In this paper, we discuss a method to compute an optimal density function that controls the diameter of ink dots by the simplex method to generate a uniform luminance on the front face BLU.

\section{Structure of BLU}

A conventional BLU for LCD display in general use for notebook computers has a structure similar to that shown in Figure 1. In this type of BLU, cold-cathode fluorescent lamp (CCFL) is used as the primary light source. The light source is located on one edge of the module and a light guide plate (LGP) is installed for light to travel from the source to the viewer.

The radiated light from the light sources are guided into a LGP by a lamp reflector. LGP is a device designed to transport light from the light source to a point at some distance with minimal loss. Light is propagated along the length of an LGP by means of total internal reflection. LGP is an optically transparent substrate usually made of polymethyl methacrylate (PMMA) and is rectangular or wedge in shape. The primary role of LGP is to extract the light in a direction perpendicualr to the direction of propagation, i.e., to a front surface of an LGP.

In conventional LGP, diffusing ink spots are printed on the back surface of the LGP. A portion of the guided light incident on the diffusing ink is reflected toward the front surface of the LGP. At the same time, small portion of the light rays that do not satisfy the condition of total internal reflection leaks out to the back and side surface of the LGP. To reuse the light by bringing back into the LGP, a reflective sheet is pasted on the back and side surface of the LGP. The emanated light over the LGP by 
dispersion from the diffusive ink is spreaded uniformly using a light-diffusing sheet, so that the viewer would not see the ink pattern on the back surface of the LGP. Two distinct prism sheets are used to collimate the light spreaded by the diffusing sheet into the direction perpendcular to the front face of an LGP, and therefore improve normal luminance.

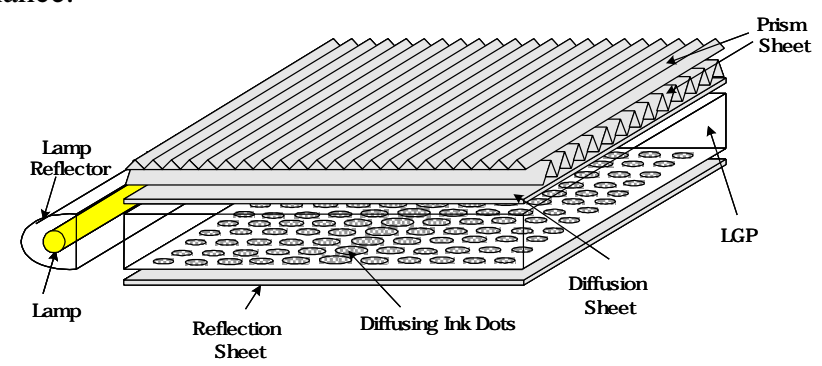

Fig. 1. A structure of conventional BLU. The lamp on the right edge of LGP is missing in this figure. The diameter of scattering ink spot increases along the light propagation direction in the LGP

To achieve a proper illumination uniformity by the emanated light on the surface of the LGP, a fill factor gradation is applied to control the size of each ink spot in an arrangement of ink spots. The size of ink spot positioned further away from the light source is relatively larger so that significant portion of the light with weak intensity because of its long propagation distance is reflected by the large ink spots.

\section{Ananysis of BLU with Monte Carlo Photon Tracing}

The design of BLU, whose goal is to maximize the luminous intensity and perfectly control the light distribution on the front face of LGP, requires the assistance of illumination design programs. The rendering techniques traditionally used in the field of computer graphics to generate synthetic images are the usual tools of choice to analyze illumination devices, like BLU. We describe an analysis method based on Monte Carlo photon tracing [3, 4] to evaluate the optical performance of BLU. The MC photon tracing simulates illumination by recursive stochastic sampling of illumination in the environment, starting with photons from the light sources.

Each photon is traced along its straight trajectory until the photon hits the nearest surface. At the photon-surface intersection position, the photon is to be reflected, transmitted, or absorbed. Whether it is reflected, transmitted, or absorbed is decided by Russian roulette [3, 4] based on the bidirectional scattering distribution function (BSDF) of the surface.

A photon is traced until it is absorbed or hits the fictitious target surface located in front of the LGP, where the target surface is associated with a regular grid. The photons passing through each bin of the grid are counted and this counter is the estimator of the equilibrium photon flux density of the position on the LGP associated with the bin. Russian roulette technique can also be applied for the termination of 
tracing individual photons. The simulation is designed to loop through successive emisisons from the source surface until a prescribed accuracy level is attained or a maximum number of photons are emitted.

\subsection{Photon Emission}

Photons are emitted at random from the surface of CCFL in this simulation model. Using random numbers, a surface location for photon emission can be selected, and then another random number can be used to assign a direction of departure for the photon. Suppose that CCFL is a cylinder with radius $r$ and length $\ell$ as shown in figure 2. Then it can be represented as a biparametric surface with each point represented by $(u, v)$ where $u$ is related to circumferential angle and $v$ is related to the length. As the surface is symmetric along the circumferentail direction and uniform along the length, uniform randum numbers $\eta_{1}, \eta_{2} \in[0,1]$ will give uniform emition point $\left(2 \pi \eta_{1}, \ell \eta_{2}\right)$ of photons on the surface of CCFL.

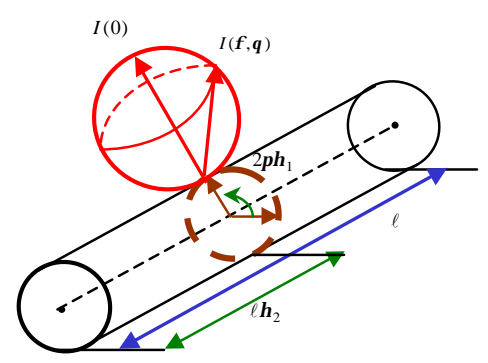

Fig. 2. Random emission of photons on the surface of CCFL

It is also assumed that CCFL emits perfectly diffuse light energy. Therefore photon emission is equally probable in all directions. The direction of photon can be represented by a spherical coordinates $(\phi, \theta)$ where $\phi$ is the circumferential angle and $\theta$ is the cone angle around the normal direction at emission point on the CCFL surface as in figure 2 . Then, these angles are sampled as:

$$
(\phi, \theta)=\left(2 \pi \xi_{\phi}, \sin ^{-1} \sqrt{\xi_{\theta}}\right)
$$

where $\xi_{\phi}, \xi_{\theta} \in[0,1]$ are uniform random numbers.

\subsection{Optical Properties of BLU components}

When a light ray is incident upon the interface between two transparent media, the incident ray is divided between a reflected ray and a refracted (transmitted) ray. The law of reflection and Snell's law predict the path of the resulting rays, and Fresnel's law of reflection predicts the amount of powers carried by each ray [8, 9]. In this 
paper, we assume that PMMA, the material of LGP, has wave-length independent radiative properties. But radiative properties of the PMMA are sensitive to direction, i.e., angle of incidence. For the angle $\theta_{i}$ of incidence, the refracted angle $\theta_{t}$ when a photon transmits to PMMA from air is calculated from the Snell's law:

$$
\eta_{a} \sin \theta_{i}=\eta_{p} \sin \theta_{t}
$$

where $\eta_{a} \approx 1.0$ is the refractive index of air, and $\eta_{p} \approx 1.49$ is the index of PMMA.

The calculations of the fractions of the incident light that are reflected or transmitted for the interface depend on polarization of the incident ray. For the light polarized perpendicular or parallel to the interface, the reflection coefficients $R_{s}, R_{p}$ are given by:

$$
R_{s}=\left\{\frac{\sin \left(\theta_{i}-\theta_{t}\right)}{\sin \left(\theta_{i}+\theta_{t}\right)}\right\}^{2}, R_{p}=\left\{\frac{\tan \left(\theta_{i}-\theta_{t}\right)}{\tan \left(\theta_{i}+\theta_{t}\right)}\right\}^{2}
$$

respectively. For the transmitted ray, the coefficient in each case is given by $T_{s}=1-R_{s}$ and $T_{p}=1-R_{p}$. For unpolarized light, the reflection coefficient becomes $R=\left(R_{s}+R_{p}\right) / 2$.

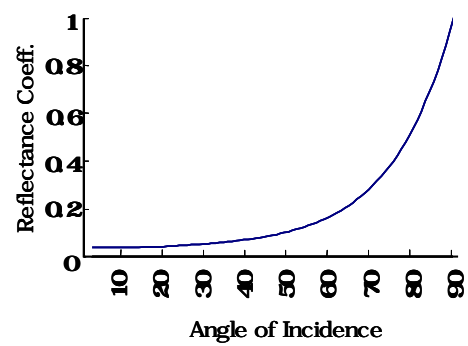

(a)

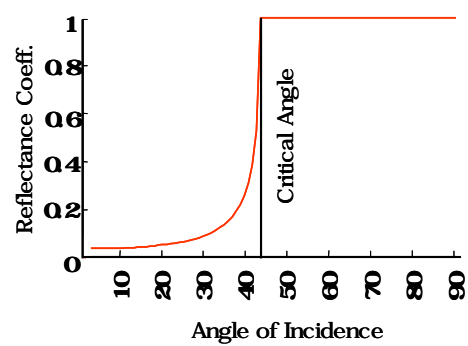

(b)

Fig. 3. Angular dependence of reflection coefficients: (a) air-to-PMMA transition (b) PMMAto-air transition

Figure 3 shows the reflectance coefficients for the air-to-PMMA and PMMA-to-air transitions, which are computed with Fresnel's equation, and they are comparable with the available experimental data. When a light moves from PMMA to air, all light is reflected if the incidence angle is above the critical angle that is $42^{\circ}$. This phenomenon is known as total internal reflection.

When a photon hits the surface of PMMA, Russian roulette is used to determine whether the photon is absorbed, reflected or transmitted [3, 4]. The surfaces of a lamp reflector and a reflection sheet are modeled with Phong model [9]. When a photon hits these surfaces, Russian roulette decides whether the photon is to be absorbed, reflected, or diffused. When the photon is reflected, the direction is importance sampled according to the BRDF of the surfaces. The surface of scattering ink dots is modeled with Lambert model. When a photon hit the surface, Russian roulette decides whether 
the photon is to be absorbed or diffused. For diffuse reflection, the direction of photon is sampled uniformly over the hemisphere as in equation (1).

\subsection{Simulation}

The fictitious target surface positioned on the front surface of LGP records the flux and the direction of the incident photons. For the following specific example, BLU for a notebook computer with 14 " LCD monitor is modeled to test the simulator. In this type of BLU, two lamp holders are mounted at the left and right edges of the LGP and two lamps are enclosed in each lamp holder. A grid of size $100 \times 100$ is associated with the target surface and the number of photons passing through each bin of the grid is counted.

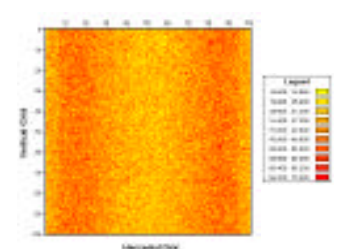

(a)

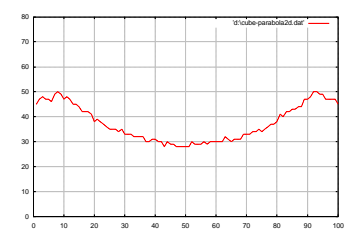

(b)

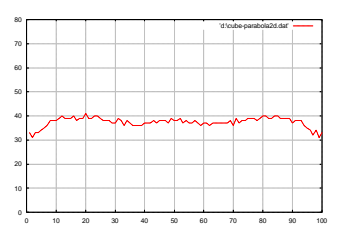

(c)

Fig. 4. The photon distribution detected on the target surface

Figure 4 (a), (b) shows a result of sample simulation, where 400,000 photons have been emitted from the source and all ink dots printed on the back surface of LGP have the same size. The count of photons in each cell of the grid is depicted in the figure 4 (a), where the lamps are positioned in the left and right sides of the grid. The result shown in figure 4 (b) corresponds to the cross section along the center line of (a). It shows high luminance on the area near to the edges and decreasing luminance getting closer to the center. Figure 4 (c) shows a cross section of a uniform illumination after optimization explained in the next section is applied to control the fill factor gradation of the ink dots.

\section{Optimization}

The purpose of ray tracing model described in previous section is to provide detailed prediction an optical performance of BLU. The performance is characterized quantitatively as a function of parameters which are defined to model the structure and optical properties of BLU components. The parameters many include the dimension of BLU components, the number and location of lamps, the shape of lamp reflector, the density (or fill factor gradation) and pattern of scattering ink dots, BSDF of scattering ink, refraction index of LGP, etc. Some parameters are assigned by fixed values during the design step and values for other parameters may change to achieve improved performance. Given an initial design of BLU by assigning some specific values to parameters modeling the structure of BLU, the ray tracing model can 
determine the performance of BLU. The next step is to analyze and enhance the design by adjusting some parameters to achieve improved performance. Numerical optimization techniques can be applied to produce an optimal design by automatically calculating the best values for the parameters.

Mathematically, the (unconstrained) optimization problem is to maximize (or, minimize) the objective function $f(\mathbf{x})$ where $\mathbf{x}=\left(x_{1}, x_{2}, \ldots, x_{n}\right)$ denotes a real $n$-vector of variables. In the ray tracing model of BLU, the variables are parameters to define the structure of BLU. The objective function is the performance of BLU, for example, the brightness and the luminance uniformity, etc. Among the parameters modeling BLU, ink pattern is considerded to be the most important factor affecting the optical performance of BLU. Optimization with respect to other parameters seems likely to be amenable to the same approach described in this paper. In this paper, we consider the problem of optimizing the ink pattern to have a uniform distribution of luminaries on the front face of BLU. The unifom distribution is measured by the root mean square (RMS) values which are the counts of photons passing through each bin of the grid associated with the target surface.

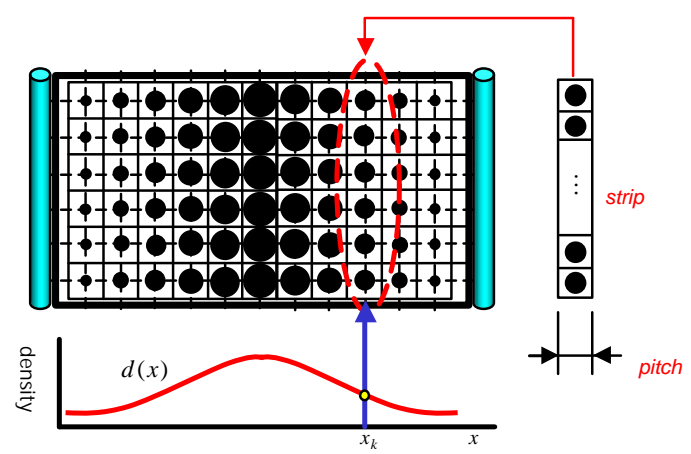

Fig. 5. The radius of circular ink dots can be computed from the density, fill factor gradation, function $d(x)$. The density function approximates the area of ink dots in a strip relative to the area of the strip, where $\mathrm{x}$ is the distance from the light source to the strip. The pitch, the width of strip, is less than $1 \mathrm{~mm}$

The ink dots are arranged in a uniform grid of square or hexagonal cells and ink spots are positioned at centers of cells, as is shown in figure 5. The cell pitch, or the distance between the centers of two adjacent cells, depend on the printing technology and a typical pitch value for current technology is less than 1 milimeter. The uniformity of the light distribution on the BLU can be obtained by varying the size of the dot per unit area of the squared grid, or the fill factor gradation of the ink spots. In general, the shape of ink dots are circles and the size of ink dots in cells of a column of sqaure cells, called a strip, in the grid is the same. Therefore the fill factor gradation can be represented by a density function $d(x)$ which approximates the area of ink dots in a strip relative to the area of the strip, where $x$ is the distance from the light source to the strip in the direction of light propagation. If a density funciton $d(x)$ is given, the 
diameter of ink dots in cells of a strip can be computed from the density function quickly.

Luminous intensity variations, as measured across the front surface of a LGP, determine the evenness of illumination. The ideal would be to have a flat illumination pattern so that the luminous intensity is the same at all points across the surface. However, in a good design there are typically minor luminous intensity variations across the surface. Careful selection of a density function design may produce an acceptable illumination pattern where the variations should not exceed $20 \%$ of the luminous intensity at center.
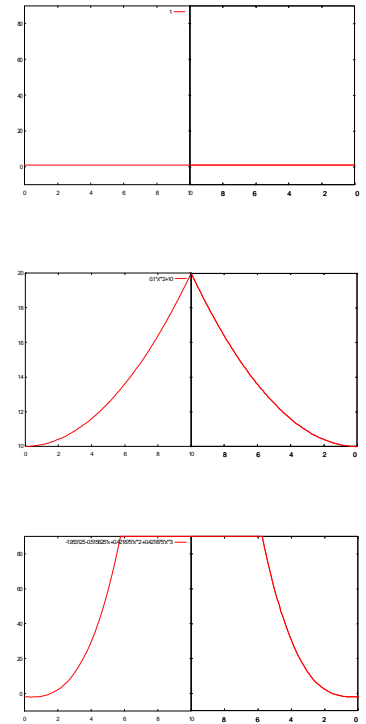

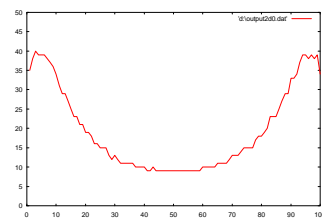

(a)

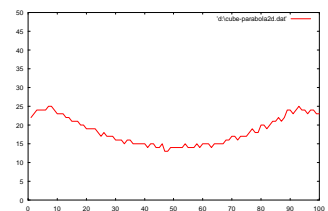

(b)

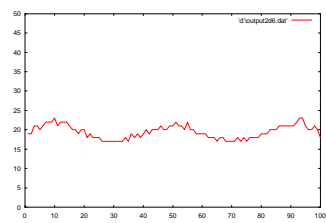

(c)

Fig. 6. Various density functions, in the left side, and the luminous intensity patterns, in the right side, that are produced by the ink patterns computed from the corresponding density functions

Figure 6 shows various density functions, in the left side, and the luminous intensity patterns, in the right side, that are produced by the ink patterns computed from the corresponding density functions. For example, figure (a) shows a luminious intensity pattern produced by a constant density function which generates very small and the same size ink dots across the grid cells. The pattern shows very low intensity in the center relative to the edges and should be avoided. Figure 6 (b), (c) show luminious intensity patterns produced by density functions which generate relatively large size ink dots in the center of the grid cells.

In this paper, we selected a polynomial of degree 3 to represent the density function. Then the objective function $f$ represents the illumination uniformity across the front face of LGP, where the parameters are four coefficients of the density function controling the size of ink dots in the cell grid. 
For the next step, optimization is needed to automatically compute the best values for the four parameters to achieve an optimal uniform illumination. We adapted the Nelder-Mead direct search method [7] to the optimal ink pattern design problem. At each iteration of the Nelder-Mead method, a simplex in $n$-dimensional space $(n=4$ in our case) is constructed, and the function $f$ in every vertex is evaluated. Based on the order of the observed function values in the vertices of the simplex, different operations can be taken to find better vertices. There are four operations on the simplex: reflection, expansion, contraction and shrinking. At each iteration, reflection, expansion, and contraction operations replace the worst vertex with the new better vertex, giving a new simplex. Shrinking operation selects the best vertex and generates new other vertices closer to the best point than previous vertices. This step is repleated until some desired bound is obtained.

Standard coefficients for operations on the simplex to control the positions of the new vertices are used in the implementation even though a delicate choice of problemdependent coefficients by some substantial level of work may improve efficiency of the implementation.

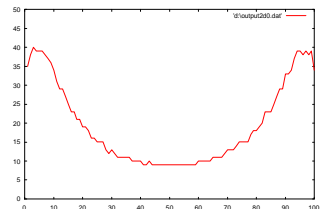

(a)

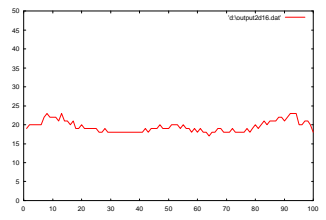

(c)

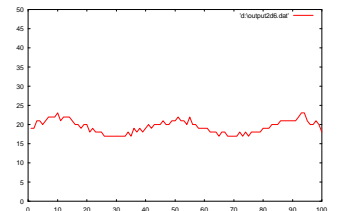

(b)

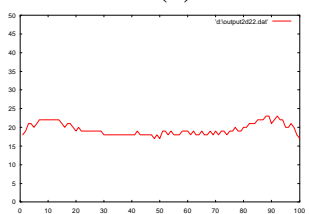

(d)

Fig. 7. Change of luminious intensity pattern generated by density function that corresponds to a simplex constructed after some iterations of the Nelder-Mead method. (a) initial density function which is a constant function (b) after $6^{\text {th }}$ iteration (c) after $16^{\text {th }}$ iteration (d) the final, after $22^{\text {nd }}$ iteration

Figure 7 shows the change of luminious intensity pattern generated by density function as the corresponding simplex rolles down to the optimal position in each iteration of the Nelder-Mead method. In this example, a constant density function is selected to assign the initial value of the four parameters of the objective function $f$.

One of the issues in the direct search method is the specification of convergence criteria. The direct search method performed well and remained popular to solve optimization problems for 30 years after it is devised in the early 1960s without a formal convergence proof. Torczon [8] discusses in detail the impossibility of developing general-purpose convergence tests for direct serach methods. In the design of BLU problem, we defined several termination conditions, for example, by assigning an appropriate value to the bound of the value $f$, on the size of simplex, etc. In a 
large number of experiments on different types of BLU, the direct search method consistently converges to an acceptable minimal solution within a reasonable number of iterations.

\section{Conclusion}

The design of BLU requires uniform illumination across the surface of BLU and high luminance enough to produce good contrast in a day environment. In this paper, an analysis method to predict the luminous intensity on the front face of BLU and an optimization method to get uniformity of illumination have been developed. The analysis method of BLU is an application of Monte Carlo photon tracing. In conventional BLU, the uniformity of illumination is controlled by the pattern of diffusing ink spots. An optimal pattern of ink dots is searched by a method based on the simplex method by Nelder and Mead.

This analysis method by Monte Carlo simulation can be applied readily to evaluate the optical performace of other strutural type of BLU. An ink pattern printed on the back surface of LGP can be replaced with micro-prism shapes grooved in the back surface of LGP. The side lighting can be replace with bottom lighting that is welcomed for large-size BLU. In the bottom lighting, the number of lamps, distances between lamps, the geometric shape of lamp reflector are very crucial to generate the uniformity of illumination and the optimal values for these parameters can be determined by the optimization technique discussed in this paper.

\section{References}

1. Gebauer, M., Benoit, P., Knoll, P., Neiger, M.: Ray Tracing Tool for Developing LCDBacklights, SID Digest 00 (2000) 558-561

2. Koyama, K.: Ray-Tracing Simulation in LCD Development, Technical Journal, Sharp Corp. (2002)

3. Pattanaik, S.N., Mudur, S.P.: Computation of Global Illumination by Monte Carlo Simulation of the Particle Model of Light. Proceedings of the $3^{\text {rd }}$ Eurographics Workshop on Rendering (1992) 71-83

4. Jensen, H.W.: Realistic Image Syntehsis Using Photon Mapping, A K Peters (2001)

5. Teijido, J.M.: Conception and Design of Illumination Light Pipes, Ph.D. Thesis, University of Geneva, Switzerland (2000)

6. Press, W.H., Flannery, B.P., Teukolsky, S.A., Vettering, W.T.: Numerical Recipes in C, Cambridge University Press, Cambridge (1988)

7. Nelder, J.A., Mead, R.: A Simplex Method for Function Minimization, Computer Journal, Vol. 7 (1965) 308-313

8. Blanis, C.A.: Advanced Engineering Electromagnetics, Jone Wiley \& Sons (1989)

9. Glassner, A.S.: Principles of Digital Image Synthesis, Morgan Kaufmann Publishers (1995)

10. Torczon, V.: On the Convergence of Pattern Search Algorithm, SIAM J. Optimization, Vol. 7 (1997) 1-25 